\title{
Comparação do efeito antiangiogênico do ranibizumab e do bevacizumab in vitro
}

\section{Comparison of anti-angiogenic effect in vitro between ranibizumab and bevacizumab}

\author{
Alexandre Cupello Souto ${ }^{1}$, Juliana Terzi Maricato ${ }^{2}$, Priscila Martins Andrade Denapoli ${ }^{1}$, Juliana Maria Ferraz Sallum ${ }^{3}$, Sang Won Han ${ }^{4}$
}

\section{RESUMO}

Objetivo: Comparar o efeito anti-angiogênico in vitro do Bevacizumab e do Ranibizumab.

Métodos: Células endotelias venosas de cordão umbilical (ECV304), cultivadas em meio $\mathrm{F} 12$ com adição de $10 \%$ de soro fetal bovino, foram plaqueadas e tratadas com concentrações clinicamente relevantes de Bevacizumab e Ranibizumab. As drogas foram administradas logo após risco realizado no meio da cultura (metodologia de scratch). Medidas lineares do espaço livre de proliferação celular foram realizadas 24, 48 e 72 horas após o momento da realização do risco. Todos os experimentos foram realizados em triplicata e a análise estatística foi feita pelo teste T-student. Resultados: $\mathrm{O}$ efeito inibitório foi observado em ambas as drogas, apenas nas concentrações 0,5 e 0,7 mg/ml. Na concentração 0,7 mg/ml, o Ranibizumab demonstrou efeito inibitório maior do que o Bevacizumab. Na mesma concentração, o Ranibizumab foi três vezes mais potente que o Bevacizumab. O efeito inibitório foi observado apenas nas primeiras 24 horas para ambas as drogas.

Conclusão: $\mathrm{O}$ Ranibizumab demonstrou efeito maior quando comparado com o Bevacizumab, porém tal efeito está mais relacionado à diferença na razão molar das drogas do que relacionada com uma diferença real no efeito anti-proliferativo.

Descritores: Neovascularização de coróide; Degeneração macular; Inibidores da angiogênese; Anticorpos monoclonais; Tomografia de coerência óptica; Angiofluoresceinografia; Injeções intravítreas; Corpo vítreo

\begin{abstract}
Purpose: To evaluate the comparative in-vitro antiangiogenic effect of Bevacizumab and Ranibizumab.

Methods: Endothelial venous umbilical cells culture (ECV304) cultivated in F12 media with addition of $10 \%$ Fetal Bovine Serum, were plaqued and treated with clinically relevant concentrations of Bevacizumab and Ranibizumab just after the scratch done in the middle of the culture (scratch methodology). Measurements of the linear size of the area free of cell proliferation were done 24,48 and 72 hours after the scratch day point. All the experiments were done in triplicate and statistical analysis were done with $T$ student test.

Results: Inhibitory effect was observed just at the concentrations of 0.5 and $0.7 \mathrm{mg} / \mathrm{ml}$ in both drugs. At $0.7 \mathrm{mg} / \mathrm{ml}$, Ranibizumab demonstrated a more potent proliferative inhibitory effect than Bevacizumab. At the same concentration, Ranibizumab was three times more potent than Ranibizumab. Inhibitory effect was observed just in the first 24 hours for both drugs.

Conclusion: Ranibizumab demonstrates an increased effect when compared to Bevacizumab and this is related more to the different molar rate of each drug than related to a real better proliferative inhibitory effect.
\end{abstract}

Keywords: Choroidal neovascularization; Macular degeneration; Angiogenesis inhibitors; Antibodies, monoclonal; Tomography, optical coherence; Fluorescein angiography; Intravitreal injections; Vitreous body

\section{INTRODUÇÃO}

Degeneração macular relacionada com a idade (DMRI) é a principal causa de cegueira legal irreversível em indivíduos acima dos 65 anos de idade em países desenvolvidos ${ }^{(1-5)}$.

A DMRI está subdividida em forma seca e úmida(3). Na forma úmida ou exsudativa ocorre o processo de angiogênese patológica com aparecimento de neovascularização coroidiana (NVC) ${ }^{(6)}$.

Este crescimento anormal dos vasos coroidianos é estimulado por citocinas, diversos fatores de crescimento incluindo o fator de crescimento vascular endotelial (VEGF), fator de crescimento do fibroblasto (FGF) e fator de crescimento derivado do epitélio pigmentado (PEDF) em resposta ao dano oxidativo ${ }^{(7)}$. A isoforma $A$ do fator de crescimento vascular endotelial (VEGF-A) tem papel importante na patogênese da NVC bem como outras doenças oculares e extraoculares que envolvam angiogênese patológica ${ }^{(8)}$. Evidências desse fato são a detecção do VEGF-A na NVC removidas de pacientes com DMRI ${ }^{(9)}$ e o aumento da expressão do VEGF-A nas células do epitélio pigmentário da retina (EPR) nos estágios iniciais da DMRI ${ }^{(10)}$. Como o VEGF se mostrou o mediador mais importante na ativação da angiogênese patológica e da permeabilidade vascular, foram desenvolvidas novas moléculas que se ligam e inibem o VEGF e seus receptores.

Bevacizumab foi o primeiro anticorpo monoclonal anti-VEGF humanizado, aprovado pelo FDA (Food and Drug Administration) americano, para utilização no tratamento de câncer de cólon metastático ${ }^{(8)}$. Em 2005, o bevacizumab foi utilizado pela primeira vez ${ }^{(11)}$ em injeção intravítrea para tratar NVC na DMRI como uso "off-label". Hoje, este tratamento tem sido amplamente aplicado(12).

Ranibizumab é o fragmento Fab de um anticorpo monoclonal recombinante que neutraliza todas as formas do VEGF-A. Foi o primeiro anticorpo anti-VEGF-A a ser aprovado pelo FDA para uso intraocular no tratamento da NVC na DMRI. Sua utilização intraocular
Submitted for publication: October 23,2010

Accepted for publication: September 22, 2011

Study carried out at the Universidade Federal de São Paulo - UNIFESP.

Physician, Centro Interdisciplinar de Terapia Gênica - CINTERGEN - Universidade Federal de São Paulo - UNIFESP - São Paulo (SP), Brazil.

2 Physician, Departamento de Microbiologia, Imunologia e Parasitologia - DMIP - Universidade Federal de São Paulo - UNIFESP - São Paulo (SP), Brazil.

3 Physician, Departamento de Oftalmologia, Universidade Federal de São Paulo - UNIFESP - São Paulo (SP), Brazil.

${ }^{4}$ Physician, Departamento de Biofísica, Universidade Federal de São Paulo - UNIFESP - São Paulo (SP), Brazil.
Funding: No specific financial support was available for this study.

Disclosure of potential conflicts of interest: A.C.Souto, None; J.T.Maricato, None; P.M.A. Denapoli, None; J.M.F.Sallum, None; S.W.Han, None.

Correspondence address: Alexandre Cupello Souto. Av. Andrade Neves, 2412 - cj 23 - Campinas (SP) 13070-001 - Brazil -E-mail: retinaldc@terra.com.br 
previne a perda visual e melhora a acuidade visual dos pacientes tratados ${ }^{(13)}$.

Como a bevacizumab e ranibizumab são drogas semelhantes é necessário avaliar se existe diferença na atividade terapêutica. Existem estudos comparativos dessas duas drogas in vitro ${ }^{(14)}$. Uma forma de medir este efeito é pela mensuração da capacidade inibitória da proliferação das células endoteliais. Neste trabalho estas drogas foram avaliadas in vitro utilizando-se uma linhagem endotelial.

O conhecimento destas informações poderão contribuir na interpretação de resultados dos estudos clínicos comparativos e na escolha da medicação a ser administrada.

\section{MÉTODOS}

\section{Cultura celular}

Foram utilizadas neste estudo células da linhagem ECV304 (Endotelial Venosa Umbilical Humana) produtoras de VEGF.

As células foram cultivadas com meio F12 contendo L-glutamina (Invitrogen), pH 7,4, suplementado com 1,4 g/l de bicarbonato de sódio e $40 \mathrm{mg} / \mathrm{ml}$ de garamicina e 10\% de soro fetal bovino. A cultura foi mantida em frascos de $25 \mathrm{~cm}^{2}$ até atingirem $80 \%$ de confluência (2-3 dias) em estufa umidificada a $37{ }^{\circ} \mathrm{C}$ e atmosfera de $5 \% \mathrm{CO}_{2}{ }^{(15)}$.

\section{Dosagem do VEGF no sobrenadante da CULtura}

Em cada poço de placa de 96 poços, $2,5 \times 10^{3}$ células da linhagem ECV produtoras de VEGF ${ }_{165}$ foram plaqueadas.

As células foram cultivadas com meio F12 contendo L-glutamina, pH 7,4 (Invitrogen), suplementado com 1,4 g/l de bicarbonato de sódio e $40 \mathrm{mg} / \mathrm{ml}$ de garamicina e 10\% de soro fetal bovino. A cultura foi mantida em frascos apropriados de $25 \mathrm{~cm}^{2}$ até atingirem $80 \%$ de confluência ( $2-3$ dias) em estufa a $370 \mathrm{C}$ e atmosfera de $5 \% \mathrm{CO}_{2}$. Em seguida o meio foi aspirado e substituído por meio fresco suplementado ou não com 10\% de soro fetal bovino. A produção basal de VEGF por essas células foi avaliada após 24, 48 e 72 horas de cultivo.

A produção de VEGF foi medida no sobrenadante das culturas por ELISA, utilizando o kit comercial DuoSet ${ }^{\circledR}$ ELISA Development System anti-VEGF humano (R\&D Systems) e seguindo as instruções do fabricante.

Este ensaio de dosagem de VEGF serviu para certificar que as células em cultura realmente produziam VEGF e para a escolha do tempo ideal de incubação das células para se aplicar as medicações nas culturas. Estas dosagens não foram utilizadas como parâmetro de avaliação da atividade antiangiogênica.

\section{Avaliação do efeito inibitório de beVACIZUmab e RANIBIZUmab}

O efeito inibitório das drogas bevacizumab e ranibizumab na cultura de células ECV304 foi avaliado utilizando-se a metodologia de "scratch". Esta metodologia consiste em fazer um risco em uma monocamada de células e medir a distância de uma borda a outra ao longo do tempo. Conforme as células se proliferam o risco tornase mais estreito e pode desaparecer completamente.

Após atingir a confluência, o meio foi retirado e a cultura lavada com $5 \mathrm{ml}$ PBS-EDTA 0,03\% para descartar possíveis células mortas no sobrenadante. As células foram então desaderidas por meio de incubação com $5 \mathrm{ml}$ de PBS-Pancreatina $0,25 \%$ a $37{ }^{\circ} \mathrm{C}$ e $5 \% \mathrm{CO}_{2}$ durante aproximadamente 5 minutos.

As células recuperadas foram centrifugadas por 5 minutos a 1500 rpm e ressuspendidas em $1 \mathrm{~mL}$ de meio F12 suplementado para contagem. A viabilidade celular foi avaliada pela adição da solução de Azul de Trypan 0,4\% em tampão fostato salino (PBS). Em seguida, as células viáveis foram contadas em câmara de Neubauer e distribuídas em placa de 96 poços. Em cada poço da placa, foram plaqueados $300 \mu \mathrm{L}$ de meio $\mathrm{F} 12$ suplementado contendo $3 \times 10^{3}$ células. A cultura foi mantida a $37^{\circ} \mathrm{C}$ em atmosfera de $5 \% \mathrm{CO}_{2}$ até atingir $80 \%$ de confluência (2-3 dias).

Em cada poço foi realizado um "risco" no meio da placa (scratch) com ponteira para micropipetas P200 (Tipak) para deixar um espaço para migração e proliferação celular. As células do sobrenadante foram removidas aspirando-se o meio de cada poço e em seguida adicionou-se meio fresco e os medicamentos como descrito a seguir.

Para testar o efeito inibitório do bevacizumab, $300 \mu \mathrm{L}$ de meio F12 suplementado contendo a droga, nas concentrações de 0,$1 ; 0,3$; 0,5 e $0,7 \mathrm{mg} / \mathrm{ml}$, foram adicionados em cada poço. O mesmo procedimento foi efetuado para avaliação do efeito inibitório do ranibizumab.

Dois grupos controle sem adição de medicamentos foram incluídos neste estudo: um grupo cultivado em meio completo e outro grupo cultivado em meio sem soro fetal bovino.

O efeito inibitório foi avaliado após 24, 48 e 72 horas a adição dos medicamentos. Todos os experimentos foram realizados em triplicatas.

\section{AQUiSIÇÃo dAS IMAGENS FOTOGRÁFICAS}

As imagens fotográficas foram capturadas utilizando a câmara Nikon (CoolPix 995) acoplada ao microscópio óptico (Nikon-TMS). Foram escolhidas imagens representativas de cada grupo experimental e fotografadas no aumento de 200x.

\section{AnÁlise estatística}

A partir de imagens capturadas, a largura do risco foi medida com uso do software Image-Pro Plus 4.0 (Media Cybernetics, L.P.). Foram realizadas três medidas por campo, gerando uma mediada média por campo. Estas medidas foram analisadas por GraphPad Prism 5.0 (GraphPad Software, Inc) utilizando o teste T-student ${ }^{(16)}$.

\section{RESULTADOS}

Primeiramente a produção de VEGF pelas células ECV304 na presença e na ausência de 10\% de soro fetal bovino (SFB) foi monitorada durante 72 horas pela dosagem pelo método de ELISA para VEGF. A produção de VEGF após 24 horas foi de $110 \mathrm{pg} / \mathrm{mL}$ e $50 \mathrm{pg} / \mathrm{mL}$ na presença e ausência de SFB, respectivamente, mostrando um estímulo de cerca de $50 \%$ de produção do fator pelo SFB (Figura 1). O acúmulo desse fator no meio de cultura aumentou na presença de SFB com máxima concentração de 400 e 780 pg/mL após 48 e 72 horas, respectivamente. Esse achado provavelmente

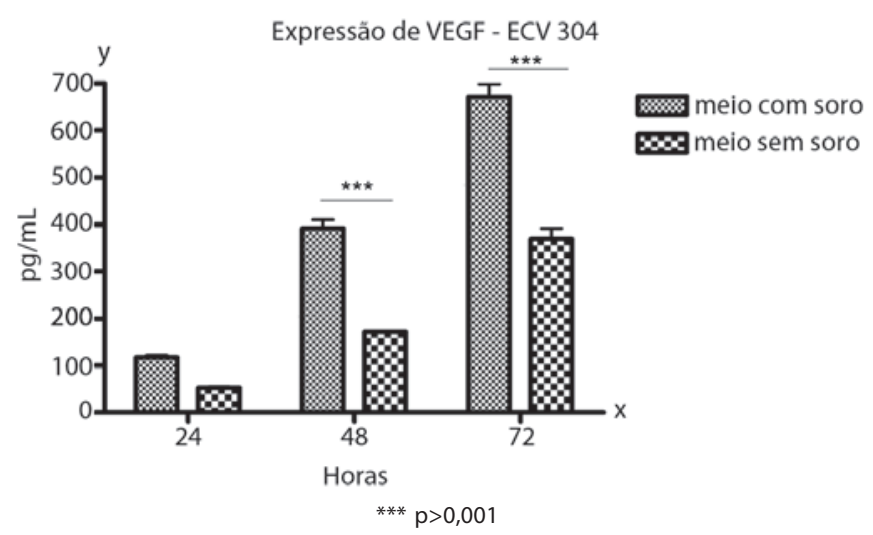

Figura 1. Avaliação da produção de VEGF pelas células ECV304. O eixo x representa os grupos de células cultivadas na presença (barra com quadriculado pequeno) ou ausência (barra com quadriculado grande) de $10 \%$ SFB e após 24,48 e 72 horas de cultivo. O eixo y representa a concentração de VEGF (dosagem realizada por ELISA) presente no sobrenadante das culturas celulares. Os resultados apresentados são valores médios de dois experimentos independentes com desvios padrões. 
deve-se à rápida multiplicação celular. Um perfil semelhante de produção de VEGF foi observado com as células mantidas na ausência de SFB, mas sempre mantendo uma relação de 50\% menor em relação à presença de SFB.

Baseando-se dos resultados da figura 1, o experimento para avaliação do efeito inibitório de bevacizumab e ranibizumab foi elaborado por meio do cultivo das células ECV304 na presença de 10\% de SFB. Para este ensaio, várias concentrações dos inibidores foram adicionadas ao meio de cultura e seus efeitos foram avaliados 24, 48 e $72 \mathrm{~h}$.

Análise microscópica está mostrada na figura 2 e as medidas quantitativas de inibição de proliferação pela análise do "scratch", na figura 3.

O efeito inibitório das duas drogas foi notado às $24 \mathrm{~h}$ a partir de $0,5 \mathrm{mg} / \mathrm{ml}$. Nos tempos maiores de incubação, nenhum efeito inibitório foi mensurável, pois já havia sido completado o repovoamento da área de "scratch" em ambas as drogas.

No caso de ranizumab, a inibição pode atingir mais de 90\% em concentrações maiores de $0,7 \mathrm{mg} / \mathrm{mL}$, enquanto que o efeito inibitório do bevacizumab sempre foi cerca de três vezes menor que ao de ranizumab.

\section{DISCUSSÃO}

A criação e o desenvolvimento do bevacizumab e do ranibizumab envolveram grande investimento. Essas drogas foram criadas com fins terapêuticos diferentes: câncer metastático do cólon no caso do bevacizumab e neovascularização coroidiana no caso do ranibizumab. Após a primeira injeção intravítrea do bevacizumab em 2005 por Rosenfeld et al..11), ambas as drogas vêm sendo amplamente utilizadas no tratamento da NVC na DMRI bem como outras doenças onde a angiogênese patológica esteja envolvida. A diferença de custo entre as duas drogas suscita discussão na comunidade oftalmológica quanto ao custo-benefício do tratamento. Tal resposta ainda não foi esclarecida. Um amplo estudo clínico comparativo está em andamento.

O bevacizumab (Genentech) foi desenvolvido por Ferrara et al., cuja publicação data de $2004^{(8)}$. Ferrara também participou no desenvolvimento do ranibizumab (Genentech, Novartis), cuja publicação data de $2006^{(17)}$
Rosenfeld demonstrou a aplicação do bevacizumab no tratamento da NVC em 2005(11) e em 2006 ${ }^{(13)}$ também demonstrou o benefício do ranibizumab no tratamento da NVC.

O ranibizumab é o fragmento Fab oriundo do mesmo anticorpo monoclonal anti-VEGF murino que originou o bevacizumab. Mais especificamente apresentam o mesmo clone do hibridoma do camundongo, conhecido como MA.4.6.1.(17), por isso apresentam ligação ao mesmo epítopo do VEGF. Do ponto de vista estrutural diferem no tamanho e peso molecular, onde o peso molecular do ranibizumab (48 kDa) é aproximadamente um terço do peso molecular do bevacizumab ${ }^{(17-19)}$. A dose para uso clínico escolhida do ranibizumab intravítreo é aproximadamente um terço da dose do bevacizumab intravítreo, provavelmente para manter a razão molar entre os dois.

No presente estudo comparativo da ação inibitória das duas drogas foi observado efeito in vitro apenas nas primeiras 24 horas pela análise do "scratch".

Nas mensurações após 48 horas, não foi observado efeito inibitório. Tal fato vem em acordo com os estudos farmacocinéticos que mostram a meia-vida curta de ambas as drogas e sugerem a necessidade do uso intraocular mais frequente. Na literatura, o uso intraocular mensal dessas drogas baseia-se em estudos matemáticos preditivos da atividade biológica(20). Foi averiguado no presente estudo que a dose/efeito inibitório foi significantemente maior $(p<0,001)$ com o ranibizumab do que o bevacizumab, nas concentrações de 0,5 e $0,7 \mathrm{mg} / \mathrm{mL}$. A razão molar entre ranibizumab e bevacizumab é de aproximadamente 1:3. Isto corrobora o fato de que mantendo-se as mesmas concentrações, foi observado efeito três vezes maior do ranibizumab quando comparado com o bevacizumab. Logo, o ranibizumab deveria teoricamente apresentar o mesmo efeito com concentração três vezes menor. Tal fato não foi observado nesse estudo, sendo condizentes com a utilização de ambas as drogas em concentrações equivalentes.

Apesar de a literatura mostrar resultados diferentes no que se refere à concentração dos medicamentos, ela mostra-se concordante com a razão molar acima descrita ${ }^{(14,18)}$. Uma possível explicação para esses achados incompatíveis pode ser a diferença metodológica, entre os estudos: diferentes tipos celulares e diferentes técnicas para avaliar o efeito inibitório das drogas.
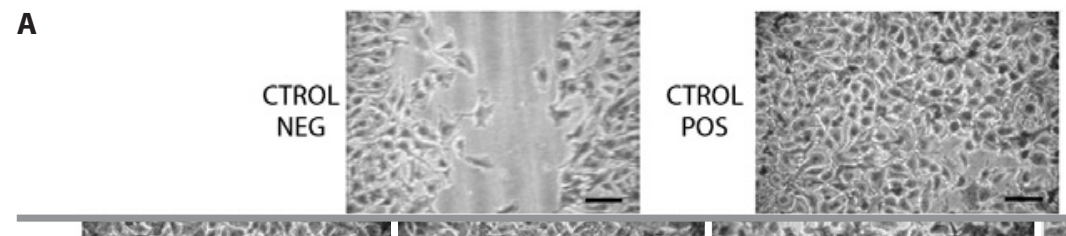

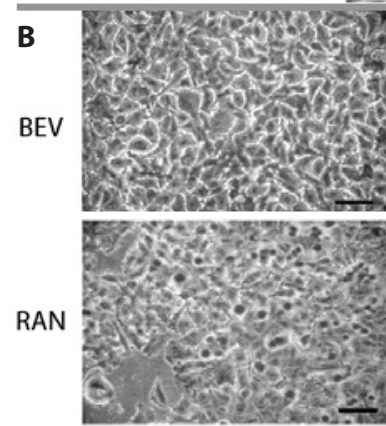

0,1

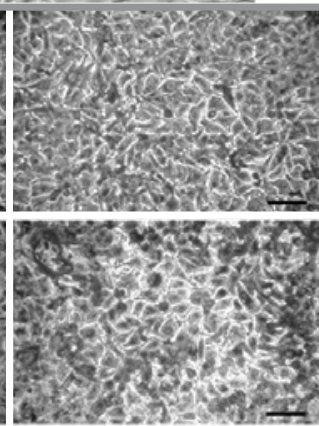

0,3

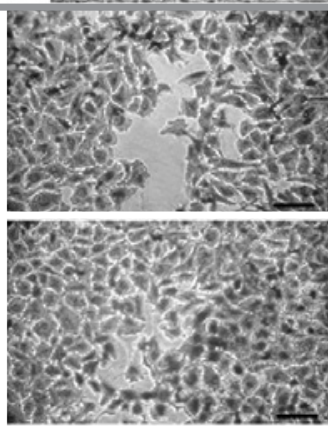

0,5

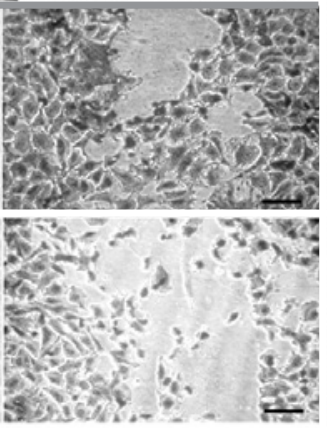

0,7

$\mathrm{mg} / \mathrm{mL}$

Figura 2. Efeito das drogas inibidoras de VEGF bevacizumab e ranibizumab na proliferação de células ECV304. As imagens foram obtidas após 24 horas de incubação com as drogas. A) Ctrl neg - controle negativo (células cultivadas sem a adição de SFB e sem a adição das drogas. Ctrl pós-controle positivo (células cultivadas com SFB e sem a adição das drogas). B) Bev - bevacizumab (células cultivadas contendo SFB e as respectivas concentrações da droga); Ran - ranibizumab (células cultivadas em meio contendo SFB e as respectivas concentrações da droga). Imagens representativas das triplicatas. As barras medem $62,5 \mu \mathrm{m}$. Foram realizados dois experimentos individuais com triplicata de cada grupo testado. 

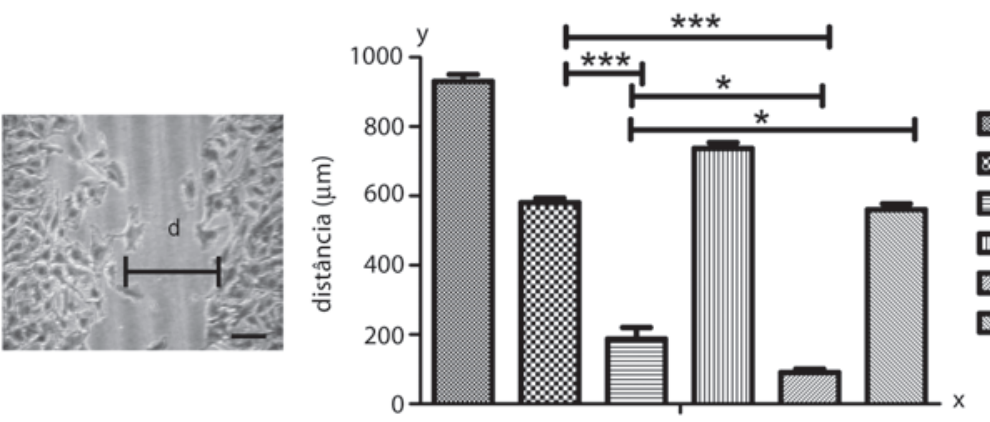

*significante $p<0,05 ;{ }^{* * *}$ muito significante $p<0,001$

Figura 3. Avaliação da ação de bevacizumab e ranibizumab na inibição da proliferação celular por meio da metodologia "scratch". A figura da esquerda é uma representação esquemática das distâncias (d) medidas no experimento. O eixo y representa os valores médios distâncias (d) para cada triplicata. As barras no eixo X representam cada grupo testado. Tempo 0 -medida do risco no início do experimento; CTR Neg - controle negativo (células cultivadas em meio F12 sem a adição de 10\% de SFB e sem a adição das drogas); Bev - bevacizumab (células cultivadas contendo SFB e as respectivas concentrações da droga); Ran - ranibizumab (células cultivadas em meio contendo SFB e as respectivas concentrações da droga). Gráfico representativo da média das medidas. Foram realizados dois experimentos individuais com triplicatas de cada grupo testado.

Existem metodologias melhores do que a técnica de "scratch" na avaliação do efeito antiangiogênico entre duas drogas. Por outro lado, o "scratch" permite avaliar o efeito migratório sem a necessidade do uso de agentes quimiotáxicos que também podem influenciar os resultados. Portanto, nesse trabalho foi avaliado o efeito inibitório das drogas principalmente pela sua interferência na migração celular, processo onde, outras moléculas além do VEGF podem estar envolvidas.

A lógica no desenvolvimento do ranibizumab foi a criação de uma molécula menor, já que estudos mostram que existe um limite de tamanho, $76 \mathrm{KDa}$, para que as moléculas se difundam através da retina(17,21). Em aparente contradição com essa ideia, estudos mostram a penetração do bevacizumab através da retina ${ }^{(22)}$.

O ranibizumab (rhuFabV2) deriva de um variante Fab humanizado, conhecido como MB1.6, oriundo do mesmo anticorpo monoclonal murino do bevacizumab. O bevacizumab por sua vez, também deriva de um variante Fab humanizado conhecido como FAB12, oriundo desse mesmo anticorpo monoclonal murino, conhecido como A.4.6.1. Posteriormente esse variante FAB-12 foi transformado em um anticorpo inteiro que ficou conhecido como bevacizumab. Procurava-se uma molécula menor para maior penetração na retina e já existia essa molécula que era o FAB-12, fragmento Fab que originou o bevacizumab, comprovadamente eficiente agente anti-VEGF. A utililização intraocular desse mesmo fragmento seria o caminho mais prático. Ao invés disso criou-se novo variante FAB do mesmo anticorpo, com novo nome, MB1.6. Esse por sua vez gerou o ranibizumab, que foi então testado no olho.

O ranibizumab contém 5 substituições de domínio variável e uma substituição de domínio constante na porção C terminal da cadeia pesada do anticorpo, quando comparado com o fragmento FAB-12 que originou o bevacizumab ${ }^{(17)}$. Teoricamente tal modificação fez o ranibizumab 5 a 20 vezes mais potente que o bevacizumab.

Acredita-se que o menor tamanho da molécula do ranibizumab seja um fator primordial para melhor difusão através da retina e consequente melhor efeito antiangiogênico (17). Outros fatores podem ser tão importantes quanto o grau de penetração dessas drogas na retina, como por exemplo, a maturação dos vasos neoformados e consequente ausência de receptores para VEGF nos vasos maduros. Além destes fatores deve ser levado em consideração a secreção contínua do VEGF, enquanto persistir o estímulo angiogênico. Desse modo, a quantidade de VEGF livre supera de maneira crescente à quantidade de anticorpo administrada. Não se deve esquecer a presença de outros fatores angiogênicos presentes além do VEGF.
Sabe-se que ambas as drogas apresentam sua maior ação antiVEGF no VEGF secretado no vítreo. Caso uma possa difundir-se mais ou menos pela retina, ambas devem chegar a quantidades não tão eficientemente terapêuticas no espaço sub-retiniano. Tal hipótese é levantada já que sua ação direta sobre a neovascularização coroidiana não tem um efeito definitivo na sua extinção. A não extinção definitiva da neovascularização coroidiana sob efeito de drogas antiVEGF como ranibizumab e bevacizumab, provavelmente está relacionada com outros fatores.

O que pode ser questionado então é o quanto a penetração na retina é uma diferença primordial entre as duas drogas.

\section{CONCLUSÃO}

Ranibizumab e bevacizumab são drogas com a mesma origem e atividade biológica. O ranibizumab demonstrou efeito maior quando comparado com o bevacizumab, porém tal efeito está mais relacionado à diferença de tamanho e à diferença na razão molar das drogas do que relacionada com uma diferença real no efeito antiproliferativo.

Resultados de estudos pré-clínicos comparativos podem auxiliar a identificar se há diferença suficiente de resultados para valer a diferença de custos envolvidos no tratamento com estas drogas.

\section{AGRADECIMENTOS}

Os autores agradecem à Dra. Helena B. Nader do Departamento de Bioquímica da UNIFESP pelas linhagens de células cedidas.

\section{REFERÊNCIAS}

1. Rosenberg T, Kile F. The incidence of registered blindness caused by age related maculopathy degeneration. Acta Ophthalmol Scand. 1996;74(4):301-402.

2. Zarbin MA. Current concepts in the pathogenesis of age related macular degeneration. Arch Ophthalmol. 2004;122(4):598-614.

3. Arroyo J. Age related macular degeneration: epidemiology, etiology and diagnosis. UpTo Date version 19.2. [cited 2005 June 16].Available from: http://www.uptodate.com/ contents/age-related-macular-degeneration-epidemiology-etiology-and-diagnosis? source $=$ search $\_$result\&search $=$age + related + macular + degeneration\&selectedTitle $=2 \% 7$ E68

4. Romani FA. [Prevalence of ocular diseases in population of elderly residents of the city of Veranopolis, Brazil]. Arq Bras Oftalmol. 2005;68(5):649-55. Portuguese.

5. Santos LP, Dinis JR, Leão AC, Sena MF. [Age related macular degeneration: analysis in two ophthalmological center in Pernambuco-Brazil]. Arq Bras Oftalmol. 2005;68(2):222-33. Portuguese.

6. Gragoudas ES, Adamis AP, Cunninghan ET Jr, Feinsod M, Guyer DR; VEGF Inhibition Study in Ocular Neovascularization Clinical Trial Group. Pegaptanib for neovascular agerelated macular degeneration. N England J Med. 2004;351(27):2805-16. Comment in: N Engl J Med. 2004;351 (27):2863-5. N Engl J Med. 2005;352(16):1720-1; author reply 1720-1. ACP J Club. 2005;143(1):18. 
7. Lois N. Neovascular age related macular degeneration. Comp Ophthalmol Update. 2004; 5:143-61.

8. Ferrara N, Hillan KJ, Gerber HP, Novotny W. Discovery and development of Bevacizumab, na anti-VEGF antibody for treating cancer. Nat Rev Drug Discov. 2004;3(5):391-400.

9. Kvanta A, Algvere PV, Berglin L, Seregard S. Subfoveal fibrovascular membranes in age-related macular degeneration express vascular endothelia growth factor. Invest Ophthalmol Vis Sci. 1996;37(9):1929-34

10. Kliffen KA, Sharma HS, Mooy CM, Kerkvliet S, de Jong PT. Increased expression of angiogenic growth factors in age-related maculopathy. Br J Ophthalmol. 1997;81 (2): 154-62.

11. Rosenfeld PJ, Moshfeghi AA, Puliafito CA. Optical coherence tomography findings after an intravitreal injection of bevacizumab (Avastin) for neovascular age-related macular degeneration. Ophthalmic Surg Lasers Imaging. 2005;36(4):331-5. Comment in: Ophthalmic Surg Lasers Imaging. 2005;36(4):270-1

12. Avery RL, Pieramici DJ, Rabena MD, Castellarin AA, Nasir MA, Giust MJ. Intravitreal bevacizumab (Avastin) for neovascular age related macular degeneration. Ophthalmology. 2006;113(3):363-72. Comment in: Ophthalmology. 2007;114(2):400; author reply 400-1.

13. Rosenfel PJ, Brown DM, Heier JS, Boyer DS, Kaiser PK, Chung CY, Kim RY; Marina Study Group. Ranibizumab for neovascular age-related macular degeneration. N England J Med. 2006;355(14):1419-31. Comment in: N Engl J Med. 2006;355(14):1409-12. N Engl J Med. 2007:356(7):748-9; author reply 749-50. N Engl J Med. 2006:355(14):1493-5.

14. Carneiro A, Falcão M, Pirraco A, Milheiro-Oliveira P, Falção-Reis F, Soares R. Comparative effects of bevacizumab, ranibizumab and pegaptanib at intravitreal dose range on endothelial cells. Exp Eye Res. 2009:88(3):522-7.
15. Moreira CR, Schmaier AH, Mahdi F, Motta G, Nader HB, Shariat-Mada Z. Identification of prolylcarboxypeptidase as the cell matrix-associated prekallikrein activator. FEBS Lett. 2002:523(1-3):167-170.

16. Choudhuri JV, Mathor MB, Silva FH, Han SW. Autonomous Growth of BALB/MK keratimocytes transfected with a retroviral vector carrying the human epidermal growth factor gene. Genet Mol Biol. 2008:31(4):824-8.

17. Ferrara N, Damico L, Shams N, Lowman H, Kim R. Development of ranibizumab, an antivascular endothelial growth factor antigen binding fragment, as therapy for neovascular age-related macular degeneration. Retina. 2006;26(8):859-70. Comment in: Retina. 2007; 27(8):1154-6; author reply 1156-8.

18. Spitzer MS, Yoeruek E, Sierra A, Wallenfels-Thilo B, Schraermeyer U, Spitzer B, et al. Comparative antiproliferative and cytotoxic profile of bevacizumab (Avastin), pegaptanib (Macugen) and ranibizumab (Lucentis) on different ocular cells. Graefes Arch Clin Exp Ophthalmol. 2007;245(12):1837-42.

19. Lowe J, Araujo J, Yang J, Reich M, Oldendorp A, Shiu V, et al. Ranizumab inhibits multiple forms of biologically active vascular endothelial growth factor in vitro and in vivo. Exp Eye Res. 2007;85(4):425-30

20. Stewart MW. Predicted biologic activity of intravitreal bevacizumab. Retina. 2007;27(9): 1196-200.

21. Jackson TL, Antcliff RJ, Hillenkamp J, Marshall J. Human retinal molecular weight exclusion limit and estimate of species variation. Invest Ophthalmol Vis Sci. 2003:44(5):2141-6.

22. Shahar J, Avery RL, Heilweil G, Barak A, Zemel E, Lewis GP, et al. Eletrophysiologic and retinal penetration studies following intravitreal injections of bevacizumab (Avastin) Retina. 2006:26(3):262-9.

\section{Congresso Internacional de Catarata e Cirurgia Refrativa}

\section{0 de maio a 2 de junho de 2012}

Parque Anhembi - SP

\section{Informações:}

CENACON - Centro Nacional de Congressos

Tel: (17) 3214-5900 - Fax: (17) 3214-5905

Site: http://www.cataratarefrativa2012.com.br 